\title{
Dielectric behavior in an oriented $\beta-P V D F$ film and chain reorientation upon transverse mechanical deformation
}

\author{
Authors: S. Lanceros-Mendez, M. V. Moreira, J. F. \\ Mano , V. Hugo Schmidt, \& G. Bohannan
}

This is an Accepted Manuscript of an article published in Ferroelectrics in 2002, available online: http://www.tandfonline.com/10.1080/00150190211756.

S. Lanceros-Mendez, M.V. Moreira, J.F. Mano, V.H. Schmidt, and G. Bohannan, "Dielectric behavior in an oriented $\beta-P V D F$ film and chain reorientation upon transverse mechanical deformation," Ferroelectrics 273, 15-20 (2002). doi: 10.1080/00150190211756

Made available through Montana State University's $\underline{\text { ScholarWorks }}$ scholarworks.montana.edu 


\title{
DIELECTRIC BEHAVIOR IN AN ORIENTED $\beta$-PVDF FILM AND CHAIN REORIENTATION UPON TRANSVERSE MECHANICAL DEFORMATION
}

\author{
S. LANCEROS-MENDEZ ${ }^{\mathrm{a}}$, M. V. MOREIRA ${ }^{\mathrm{a}}$, J.F. MANO ${ }^{\mathrm{b}}$, \\ V. H. SCHMIDT ${ }^{\mathrm{c}}$ and G. BOHANNAN ${ }^{\mathrm{c}}$ \\ ${ }^{2}$ Dept. de Física, Universidade do Minho, 4710-057 Braga, Portugal \\ bept. of Polymer Eng., Univ. of Minho, 4800-058 Guimarães, Portugal \\ 'Dept. of Physics, Montana State University, Bozeman, MT 59717, \\ USA
}

(Received in final form November 24, 2001)

Films of semicrystalline poly(vinylidene fluoride) (PVDF) in the $\beta$ phase have been studied by means of dielectric measurements and Fourier Transform Infrared Spectroscopy (FTIR). The main goal of the study was to compare the dielectric relaxations of $\alpha$ - and $\beta$-PVDF and to improve the understanding of the structural changes that occur in $\beta$ PVDF during a mechanical deformation process and their impact in the electromechanical properties of the polymer. A reorientation of the chains and a decrease in the degree of crystallinity with increasing deformation was observed.

Keywords: PVDF; dielectric; infrared; plastic deformation; relaxation

\section{INTRODUCTION}

The piezoelectric polymer polyvinylidene fluoride (PVDF) has been intensely investigated because of its interesting properties and increasing technological applications. PVDF can exist in several crystalline phases ${ }^{[1]}$. Of the five known modifications, the $\alpha$ - and $\beta$ phases are most common. The $\alpha$-form arises usually from the melt when PVDF crystallizes in quiescent conditions. The $\beta$-form offers the highest piezo, pyro- and ferroelectric properties. In order to obtain $\beta$ PVDF a technological process involving stretching and poling of extruded thin sheets of the polymer is used, obtaining permanent polarization $^{[2]}$. $\beta$-PVDF presents different properties than $\alpha-P V D F$. In this work the relaxation behavior of $\beta$-PVDF is investigated with dielectric spectroscopy; such data are compared with the results reported for $\alpha$-PVDF. 
Deformation processes are not only intimately associated with the production and use of these films: the oriented films are often subjected to further stresses during service and morphological changes may occur mainly in the direction perpendicular to the preferred orientation of the chains, where the mechanical properties are weaker.

In this study a set of experiments were carried out in $\beta$-PVDF in order to improve our knowledge on the structural and dipolar changes that occur in this material when subjected to a deformation process at room temperature.

\section{EXPERIMENTAL}

The starting material was a $28 \mu \mathrm{m}$ thick commercial $\beta$-PVDF film from Measurements Specialties, Inc. (Fairfield, NJ, USA).

Dielectric measurements were performed with a General Radio 1693 digital RLC bridge. The real $\left(\varepsilon^{\prime}\right)$ and imaginary $\left(\varepsilon^{\prime \prime}\right)$ parts of the permittivity were obtained in the frequency range $100 \mathrm{~Hz}$ to $100 \mathrm{kHz}$ at a temperature rate of $1{ }^{\circ} \mathrm{C} \cdot \mathrm{min}^{-1}$ from $-60^{\circ} \mathrm{C}$ to $140^{\circ} \mathrm{C}$. The calibration of temperature was carried out according to $^{[3]}$.

Tensile tests were carried out at room temperature in an Instron 4505 universal testing machine at test velocities of $\mathrm{d} l / \mathrm{d} t=2 \mathrm{~mm} / \mathrm{min}$ in $8 \mathrm{~cm} \times 1 \mathrm{~cm}$ rectangular samples. Some samples were prepared by controlled deformation to different degrees (from 18\% to break (max. $140 \%)$ ) in the perpendicular direction to the initial chain orientation ${ }^{[4]}$.

Infrared spectra were obtained in transmission mode at room temperature on a BRUKER $66 \mathrm{~V}$ spectrometer from 400 to $6000 \mathrm{~cm}^{-1}$ using polarised light in perpendicular $(\perp)$ and parallel $(\|)$ modes, with a resolution of $1 \mathrm{~cm}^{-1}$.

\section{RESULTS AND DISCUSSION}

\section{a) Dielectric Measurements}

Dielectric results on the studied $\beta$-PVDF film show the low temperature $\beta$-relaxation, assigned to the glass transition dynamics, but no $\alpha$-relaxation is observed at higher temperatures, associated with molecular motions within the crystalline fraction. An increase of the dielectric loss and a shrinking of the sample are observed instead. The release of the stress that was locked in when the film was originally stretched causes the shrinkage into an unrestrained film. We observed a decrease of $\sim 45 \%$ along the stretch direction at $130{ }^{\circ} \mathrm{C}$. So, the decrease in $\varepsilon^{\prime}$ above $90^{\circ} \mathrm{C}$ is associated with the change in the geometry of the sample owing to release of the molecular orientation. It is interesting to 
note that the $\alpha$-relaxation, associated with molecular mobility within the crystalline phase was observed in $\alpha$-PVDF ${ }^{[5]}$.

The dynamic glass transition was analysed by the Vogel-TammannFulcher relaxation formalism

$$
\tau=\tau_{0} \exp \left(\mathrm{E}_{\mathrm{VTF}} / \mathrm{k}\left(\mathrm{T}-\mathrm{T}_{0}\right)\right),
$$

where $\mathrm{E}$ is the VTF energy and $\mathrm{T}_{0}$ is the critical temperature at which molecular motions in the material become infinitely slow. The parameters obtained were $\tau_{0}=0.58 \times 10^{-11} \mathrm{~s}, \mathrm{~T}_{0}=196.54 \mathrm{~K}$ and $\mathrm{E}_{\mathrm{VTF}}=0.061$ $\mathrm{eV}$ (Figure 1). Comparing to the results obtained for the same relaxation in $\alpha$-PVDF $\left(E_{\mathrm{VTF}}=0.088 \mathrm{eV}, \tau_{0}=1.2 \times 10^{-11} \mathrm{~s}, \mathrm{~T}_{0}=187.5 \mathrm{~K}\right)$ we observe a decrease in the VTF energy and $\mathfrak{t}_{\overline{0}}$ and an increase in $\mathrm{T}_{0}{ }^{[6]}$.
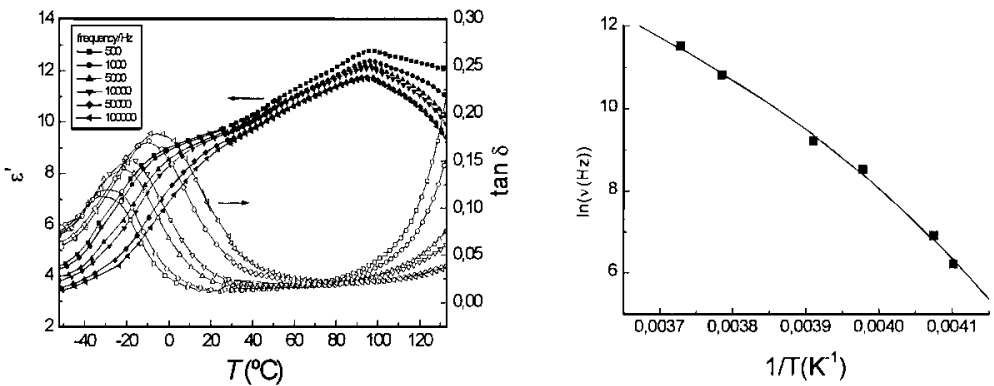

FIGURE 1 Dielectric permittivity measured for $\beta$-PVDF between $-60 \mathrm{C}$ and $130 \mathrm{C}$ at several frequencies (left). Vogel-TammannFulcher fit of the $\beta$-relaxation of $\beta$-PVDF (right).

A consequence of the values of the fitting parameters of the VTF relaxation plot is the calculation of the fragility parameter ${ }^{[7]}$

$$
m=\frac{E_{V T F} / k T_{g}}{(\ln 10)\left(1-T_{0} / T_{g}\right)^{2}}
$$

where $m$ is an indication of the steepness of the variation of the material properties (viscosity, relaxation time...) as $T_{g}$ is reached. A high $m$ value defines a fragile material whereas a strong one will be characterized by small $m$ values.

The values of several glass-forming amorphous polymers range from 46 (polysobutylene) to 191 (polyvinylchloride) ${ }^{[7]}$. The $m\left(\mathrm{~T}_{\mathrm{g}}\right)$ value calculated with the VTF parameters for $\beta$-PVDF is 92 determined at the 
glass transition temperature where the relaxation time is equal to $100 \mathrm{~s}$. The value for $\alpha$-PVDF is 87 . The fragility parameters of $\alpha$ - and $\beta$ PVDF are very similar. This demonstrates that only the amorphous part of the material is involved in the relaxation process. The fragility parameter indicates that PVDF is not a very fragile material compared with the polymers analyzed in ${ }^{[7]}$. The low fragility can be associated to the semicrystalline nature of this polymer ${ }^{[8]}$.

\section{b) Mechanical tests}

The anisotropy of the original $\beta$-PVDF films is observed in the stressstrain mechanical experiments carried out in the two main directions (parallel and perpendicular to the polymeric chains). In the longitudinal direction the film shows a typical brittle behaviour with a higher ultimate stress and a lower strain at break than for the transversal direction, in which the yielding point is reached ${ }^{[2,4]}$.

\section{c) Infrared Spectroscopy}

Samples with different levels of deformations within the plastic region (from 15\% to break (max. 140\%) were prepared and studied by FTIR and $\mathrm{DSC}^{[4]}$. For deformations above $35 \%$, the FTIR spectra of the deformed samples for a given polarisation seemed to be identical to the spectra of the non-deformed samples in the other polarisation ${ }^{[4]}$, i.e. a polarisation switching seemed to occur (Figure 2). It was suggested that the inversion of polarisation possibly occurs near the yield point of the specimen $\mathrm{s}^{[4]}$. A more detailed investigation of the stress-recovering region (deformations between $\sim 15 \%$ and $\sim 30 \%$ ) shows that the polarization switching occurs progressively within this region: in Figure 2 can be observed that whereas the sample with a deformation of $18 \%$ (right after the yielding point) shows a mode structure similar to the one of the undeformed sample and in the $40 \%$ deformed sample the switch seems to be already completed, the sample with a deformation of $30 \%$ shows a mode structure intermediate between this two conformations.

Such inversion of behaviour from longitudinal to transversal direction, and vice-versa, suggests changes of morphologies within the crystalline fraction such that the chains in the crystalline phase switch their direction by $90^{\circ}$. This inversion of the spectra seems to occur continuously from the yielding point along the stress-recovering region i.e., in the early plastic region (Figure 2, right). Further, with increasing of strain the changes are less pronounced (Figure 2) ${ }^{[4]}$ 

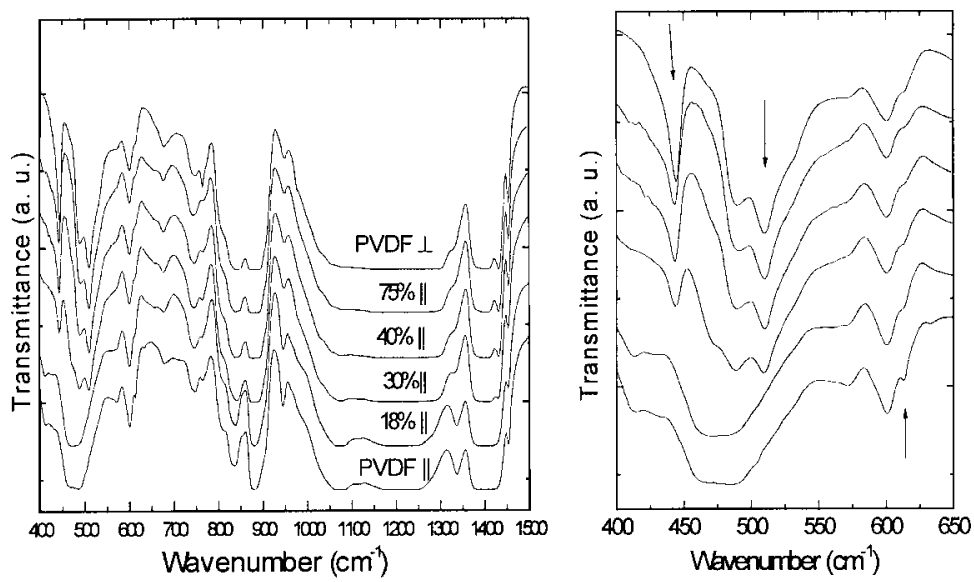

FIGURE 2 Left: Comparison of the FTIR spectra for $\beta$ PVDF with four different deformations $(18 \%, 30 \%, 40 \%$ and $75 \%$ ) in parallel mode with a virgin sample in both polarisations. Right: detail of the $400 \mathrm{~cm}^{-1}$ to $650 \mathrm{~cm}^{-1}$ spectral region.

The result suggests that yielding and further plastic drawing induce a complete reorientation of the crystal lamellae. Morphological changes in semicrystalline polymers during stress application have been studied by Flory ${ }^{[9]}$ and Takahashy and co-workers ${ }^{[10]}$ who proposed that plastic deformation occurs by fusion and recrystallisation of the crystal phase present in the semicrystalline polymer. This model was used to explain a local $90^{\circ}$ reorientation of the polymeric segments in the crystalline structure of PVDF ${ }^{[1]}$ as seen by creep and stress-strain experiments.

This reorientation leads to a dipolar orientation and to a change in the electromechanical properties of the strained material. The actual dipolar reorientation during straining the material is demonstrated by the curves of infrared transmittance versus strain. The realignment of the dipoles in $\beta$-PVDF with a poling field was similarly evidenced by the variation of the infrared transmittance at $512 \mathrm{~cm}^{-1}$, which is associated with vibration along the direction of the $\mathrm{CF}_{2}$ dipole vector ${ }^{[12]}$

Differential scanning calorimetry (DSC) performed in the previously deformed films did not exhibit different crystalline phases. Further, the film deformation changes the degree of crystallinity of PVDF: the crystallinity of the samples decreases linearly from $47.9 \%$ 
in the non-deformed samples to $35.8 \%$ in the samples deformed $140 \%{ }^{[4]}$. On the other hand, the strong changes in the morphology during the film drawing are not accompanied by changes in the crystalline lamellae thickness ${ }^{[4]}$.

\section{CONCLUSION}

Films of semicrystalline poly(vinylidene fluoride) (PVDF) in the $\beta$ phase have been studied by means of dielectric measurements and Fourier Transform Infrared Spectroscopy (FTIR). Especial attention was paid to the modification occurring during the deformation process. FTIR spectra show a reorientation of the chains from perpendicular to parallel to the stress orientation within the plastic region. A decrease in the degree of crystallinity due to the deformation is observed. On the other hand, the thickness of the lamellae seems to remains practically unchanged.

\section{ACKNOWLEDGMENTS}

Work partially supported by Fundação para a Ciência e Tecnologia (PRAXIS/P/CTM/14171/1998 and POCTI/1999/CTM/33501) and by a NSF grant (DMR-9520251).

\section{REFERENCES}

[1.] H. S. Nalwa, J. Macromol. Sci, C31(4), 341-432 (1991)

[2.] A. Vinogradov, F. Holloway, Ferroelectrics, 226, 169.-181 (1999)

[3.] J.F. Mano, S. Lanceros-Méndez, A.M. Nunes, M. Dionísio, J. Thermal Analys. and Calorim. 65, 37 (2001)

[4.] S. Lanceros-Méndez, J. F. Mano, A. M. Costa, and V. H. Schmidt, to be published in J. Macromol. Sci., 2001

[5.] J.W. Sy, J. Mikovic, Macromolecules 2000, 33, 933

[6.] M. Grimau, E. Laredo, A. Bello, N. Suarez, J. Polym. Sci. B: Polym Phys, 35, 2483 (1997)

[7.] R. Bohmer, K.L. Ngai, C.A. Angell and D.J. Plazek, J. Chem. Phys. 99, $4201(1993)$

[8.] A. Bello, E. Laredo, M. Grimau, Phys. Rev. B, 60, 12764 (1999)

[9.] P. J. Flory, D. Y. Yoon, Nature, 272, 226 (1978).

[10.] Y.Takahashy, T. Zakoh, N. Hanatani, Colloid Polym. Sci. 269, 781 (1991)

[11.] S. Hellinckx; J.-C. Bauwens, Colloid Polym Sci. 273, 219 (1995)

[12.] D. Naegele and D. Y. Yoon, Appl. Phys. Lett. 33, 132 (1978) 